\title{
Frequency and Temperature Dependences of Dielectric Dispersion and Electrical Properties of Polyvinylidene Fluoride/Expanded Graphite Composites
}

\author{
Yu Chao Li, Robert Kwok Yiu Li, and Sie Chin Tjong \\ Department of Physics and Materials Science, City University of Hong Kong, Kowloon Town, Hong Kong \\ Correspondence should be addressed to Sie Chin Tjong, aptjong@cityu.edu.hk
}

Received 19 June 2009; Accepted 21 October 2009

Academic Editor: Gaurav Mago

Copyright (c) $2010 \mathrm{Yu}$ Chao Li et al. This is an open access article distributed under the Creative Commons Attribution License, which permits unrestricted use, distribution, and reproduction in any medium, provided the original work is properly cited.

\begin{abstract}
A conductive nanofiller, expanded graphite (EG), was introduced into polyvinylidene fluoride (PVDF) by direct melt blending process. The electrical conductivity and dielectric properties of resulting PVDF/EG composites were investigated in a wide range of frequencies from $10^{2}$ to $10^{8} \mathrm{~Hz}$. The dielectric dispersion and conductivity of the composite system exhibited a strong frequency dependence particularly in the vicinity of percolation threshold $\left(\Phi_{c}=6 \mathrm{vol} \%\right)$. Moreover, the dielectric permittivity tended to decrease with increasing frequency while the conductivity displayed a reverse trend. A large permittivity was found in the composite having EG content near $\Phi_{c}$. Accordingly, percolation concept and biased random walk approach can be used to describe the carrier transport of percolating PVDF/EG system. The conductivity and permittivity of percolating PVDF/EG composites were observed to be temperature dependent.
\end{abstract}

\section{Introduction}

Conductive polymer composites reinforced with carbon black (CB) microparticles have been used extensively in industrial sectors as materials for antistatic and electromagnetic shielding. Such polymer microcomposites generally require a large filler concentration to achieve desired electrical properties. The additions of large filler content to polymers lead to poor processability and inferior mechanical properties. In contrast, polymer nanocomposites reinforced with expanded graphite (EG) platelets have shown substantial improvements in electrical conductivity and mechanical property over microcomposites reinforced with CB [1].

Graphite is a carbonaceous material available commercially at relatively low cost. Its structure consists of carbon layers in an alternating stacked sequence. These layers are bonded by weak van der Waals forces. Consequently, oxidizing agent such as sulfuric acid can be easily intercalated into graphite interlayers, forming the so-called graphite intercalation compound (GIC). Expanded graphite (EG) with a high aspect ratio and excellent electrical conductivity can be produced by exfoliating GIC through rapid heating in a furnace or microwave environment. EG nanoplatelets have been successfully incorporated into polyamide- 6 , poly(methylmethacrylate), polystyrene and polyolefins to improve their thermal stability, electrical and mechanical performances [2-4].

Polyvinylidene fluoride (PVDF) is a semi-crystalline thermoplastic having remarkable high piezoelectric coefficient $[5,6]$, excellent thermal stability and chemical resistance compared with other polymers. Consequently, PVDF and its copolymers find widespread industrial applications in transducers, transistor and capacitors. Moreover, PVDF is an attractive polymer matrix for micro- and nanocomposites with superior mechanical and electrical properties [7-11]. Very recently, Almasri et al. [11] investigated the microstructure, electrical and thermomechanical behaviors of solution cast PVDF composites reinforced with carbon nanotubes (CNTs). Their results show that the double-walled CNTs act as nucleation sites for PVDF polymer chains during solution processing. Electrical measurement revealed that the PVDF/CNT nanocomposites exhibit a low percolation threshold. The low percolation filler concentration for electrical conductivity is resulted from very high aspect ratio 
TABle 1: Physical properties of PVDF (Kynar 740).

\begin{tabular}{lc}
\hline Physical properties & Value \\
\hline Density & $1.78 \mathrm{~g} / \mathrm{cm}^{3}$ \\
Melting flow rate & $1.1 \mathrm{~g} / 10 \mathrm{~min}\left(230^{\circ} \mathrm{C} / 5 \mathrm{~kg}\right.$ load $)$ \\
Melting point & $168^{\circ} \mathrm{C}$ \\
Electrical resistivity & $2 \times 10^{14} \mathrm{ohm} . \mathrm{cm}$ \\
Dielectric constant & 7.70 under $10^{6} \mathrm{~Hz}$ \\
& 10.8 under $100 \mathrm{~Hz}$ \\
\hline
\end{tabular}

(above 1000) of CNTs $[12,13]$. However, the high cost of CNTs precludes their extensive application as nanofillers for polymers. In contrast, graphite is naturally abundant and available at a relatively low cost. Graphite nanoplatelets also exhibit good electrical conductivity $\left(\sim 10^{4} \mathrm{~S} / \mathrm{cm}\right)$, thus are ideal nanofillers for conducting polymer nanocomposites. The aspect ratio of graphite nanoplatelets $(\sim 250)$ is smaller than that of CNTs, but conducting filler network can also be established in the polymer/EG nanocomposites. This implies that the polymer/EG nanocomposites only require low EG loadings to achieve high electrical conductivity [14]. In terms of economic consideration, it is more cost effective to use EG nanoplatelets to prepare PVDF/EG nanocomposites. However, little is known on the PVDF/EG nanocomposites as far as we know. Accordingly, we prepared PVDF/EG nanocomposites via melt blending and investigated their electrical behavior at various frequencies and temperatures in details. The results are discussed and compared with existing models. The fabrication, microstructure and thermal properties of PVDF/EG nanocomomposites have been reported elsewhere [15].

\section{Experimental}

2.1. Materials. Commercial grade of PVDF (Kynar 740) was supplied by Atofina Chemicals Inc., USA. The physical properties of the matrix material are shown in Table 1. Expandable graphite flake (GIC, 220-50N) was purchased from GrafTech Ltd., USA. EG was obtained via simple thermal-shock treatment of the as-received GIC in an oven at $700^{\circ} \mathrm{C}$ for 10 seconds. The obtained EG maintains excellent electric conductivity $\left(\sim 10^{4} \mathrm{~S} \cdot \mathrm{cm}^{-1}\right)$ similar to natural graphite. Figures $1(\mathrm{a})-1(\mathrm{~d})$ show SEM images of expandable graphite (Figures 1(a) and 1(b)) and resulting expanded graphite (Figures 1(c) and 1(d)), respectively. The expandable graphite commonly known as the graphite intercalation compound (GIC) comprises of stacked graphene layers intercalated with acid reagents. The incorporation of intercalants at graphite galleries leads to an expansion of the graphite lattice (Figure 2) [16]. From Figure 1(c), EG appears as a loose, porous or worm-like morphology having numerous entangled nanosheets. The thickness of individual graphite sheet determined from the SEM micrograph at higher magnification is $\sim 50 \mathrm{~nm}$ (Figure 1d).

2.2. Fabrication of Nanocomposites. The fabrication procedure could be seen in our previous study [16]. Briefly,
PVDF/EG nanocomposites were compounded in an internal mixer (Haake, Rheomix 600) at $220^{\circ} \mathrm{C}$ under $80 \mathrm{rpm}$ for 15 minutes. The EG contents of nanocomposites were fixed at $2,4,6,8,10,12,14 \mathrm{wt} \%$, corresponding to volume concentration of $1.56,3.14,4.73,6.33,7.95,9.59$ and $11.2 \%$, respectively. The volume content $\left(\Phi_{\mathrm{vol}}\right)$ of EG can be determined from the following equation [15]:

$$
\phi_{\mathrm{vol}}=\frac{\phi_{\mathrm{wt}} / \rho_{\mathrm{EG}}}{\phi_{\mathrm{wt}} / \rho_{\mathrm{EG}}+\left(1-\phi_{\mathrm{wt}}\right) / \rho_{\mathrm{PVDF}}} \times 100 \%,
$$

where $\Phi_{\mathrm{wt}}$ represents the EG weight concentration, $\rho_{\mathrm{EG}}$ and $\rho_{\text {PVDF }}$ are the density of EG $\left(2.29 \mathrm{~g} / \mathrm{cm}^{3}\right)$ and PVDF $\left(1.78 \mathrm{~g} / \mathrm{cm}^{3}\right)$, respectively. Although EG exhibits a loose, porous structure, its density is assumed closer to that of natural graphite $\left(2.25 \sim 2.30 \mathrm{~g} / \mathrm{cm}^{3}\right)$. The resulting composites were compression-molded at $230^{\circ} \mathrm{C}$ under $20 \mathrm{MPa}$ for 10 minutes. Disk samples with diameter of $12 \mathrm{~mm}$ were punched from these molded plates.

2.3. Electrical Measurements. The disk specimens were polished and then coated with silver paste at both sides as two electrodes. AC electrical conductance and capacitance were measured using an impedance analyzer (Agilent model 4294) from frequency $10^{2}$ to $10^{8} \mathrm{~Hz}$ at room temperature. The impedance analyzer was equipped with a small furnace for high temperature measurements. The heating rate of the furnace was maintained at $5^{\circ} \mathrm{C} / \mathrm{min}$.

\section{Results and Discussion}

3.1. AC Electrical Properties. Figures 3(a)-3(c) display the frequency dependence of electrical properties. The variation of $\sigma$ (AC conductivity) with frequency for different EG content of composites is shown in Figure 3(a) at room temperature. At low EG contents $(\leq 6 \mathrm{wt} \%)$, the electrical conductivity of the nanocomposites increases with increasing frequency. These specimens show a typical insulating behavior with a frequency-dependent conductivity. When the EG content reaches $8 \mathrm{wt} \%$, there is a transition from an insulator to semiconductor. The transition filler concentration of the composite from insulating to conducting is defined as percolation threshold $\left(\Phi_{c}\right)$ associated with the formation of conducting network. The insulator-conductor transition is also confirmed by the large dissipation factor $(\tan \delta$ ) for PVDF/8 wt\% EG composite (Figure 3(b)). Above $\Phi_{c}$, the AC conductivity remains nearly unchanged in the lower frequency region, but becomes frequency dependent approaching the onset frequency $\left(f_{c}\right)$. In this case, the composites display a conducting characteristic over entire range of frequency studied. The frequency independent conductivity is commonly regarded as DC conductivity.

The variations of $\varepsilon^{\prime}$ (the real part of the complex dielectric permittivity: $\varepsilon^{*}=\varepsilon^{\prime}-j \varepsilon^{\prime \prime}$ ) PVDF/EG nanocomposites as a function of frequencies at room temperature is shown in Figure 3(c). As expected, the variation tendency of dielectric constant with frequency is the reverse of electrical conductivity. The $\varepsilon^{\prime}$ attains high value at low frequency and decreases exponentially with increase in frequency. The decrease of 


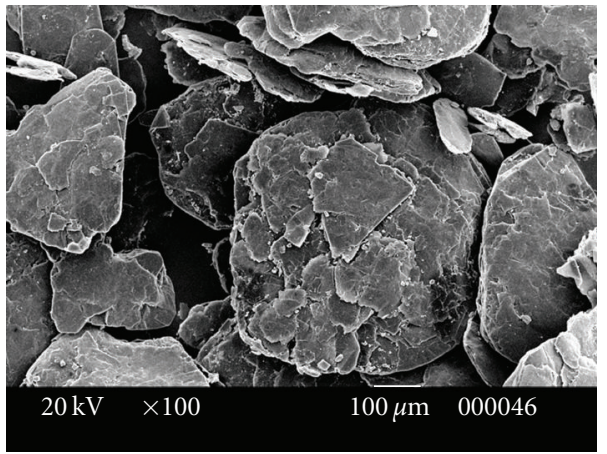

(a)

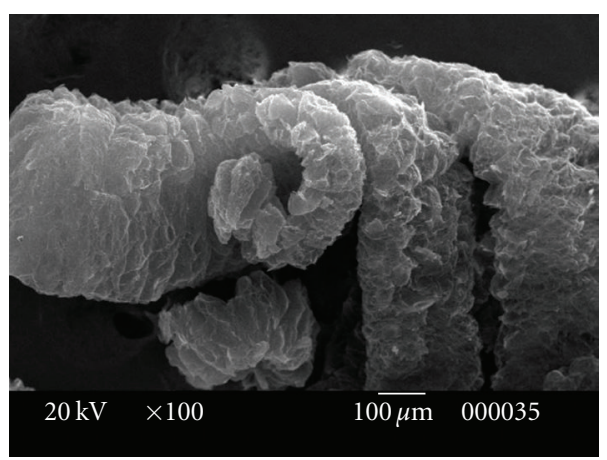

(c)

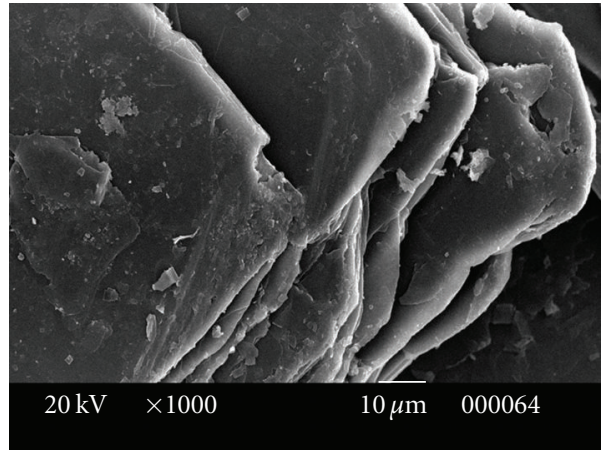

(b)

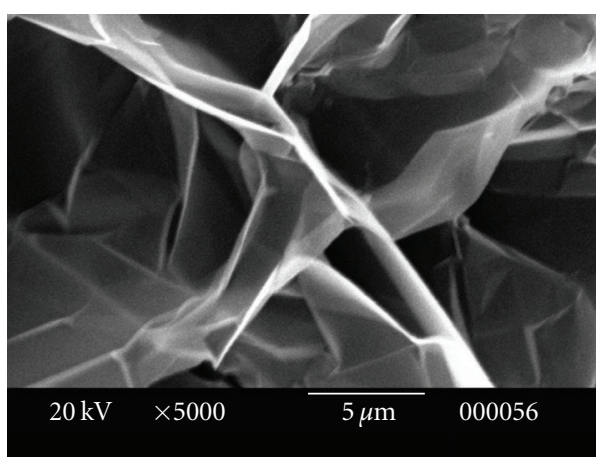

(d)

FIgURE 1: SEM images of expandable graphite (a), (b) and expanded graphite (c), (d).

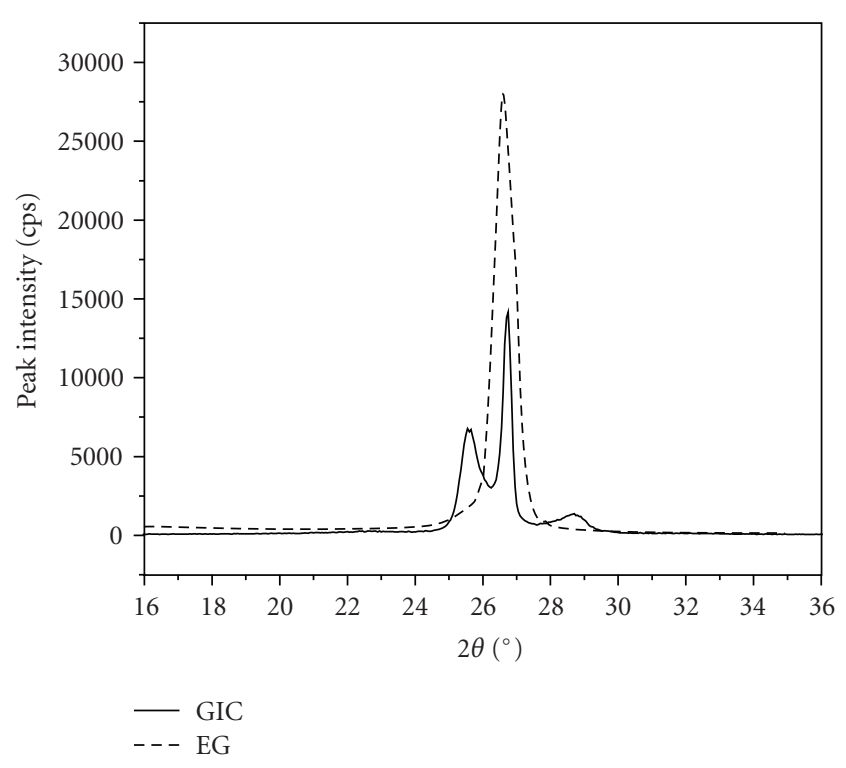

FIGURE 2: XRD patterns of expandable graphite (GIC, Solid line) and expanded graphite (EG, dashed line).

dielectric constant is mainly attributed to the mis-match of interfacial polarization of composites to external electric field at elevated frequencies [17]. The permittivity increases dramatically near the percolation threshold. It is usually believed that the percolation threshold is an important point at which electrical property varies a lot. Therefore, study conducting composites in the vicinity of percolation threshold is an effective way to know the electrical transport behavior of composites.

According to the percolation theory [18], the variations of $\sigma(f)$ and $\varepsilon^{\prime}(f)$ with frequency follows a power law as the EG content approaches percolation threshold:

$$
\begin{gathered}
\sigma\left(f, \phi_{c}\right) \propto \omega^{u} \\
\varepsilon^{\prime}\left(f, \phi_{c}\right) \propto \omega^{-v},
\end{gathered}
$$

where $\omega=2 \pi f$ is the angular frequency, $u, v$ are critical exponents and theoretically in $u+v=1$. In practice, the $\mathrm{AC}$ conductivity response for various materials can be determined by two parts mathematically as expressed in the following equation [19-21]:

$$
\sigma(f)=\sigma_{\mathrm{DC}}+A \omega^{u}
$$

where $\sigma_{\mathrm{DC}}$ is DC conductivity of composites, $A$ is a temperature dependant constant. Equation (4) is often called "AC universal dynamic response" [21] or "AC universality law" [22] because various materials display such behavior. Therefore, AC conductivity can be recognized as the combined effect of DC conductivity $(f=0 \mathrm{~Hz})$ caused by migrating charge carriers and frequency induced dielectric dispersion. In this case, a large DC conductivity caused by formation of conducting path-way significantly dominates the transport behavior in a broad frequency range as seen the plateau 


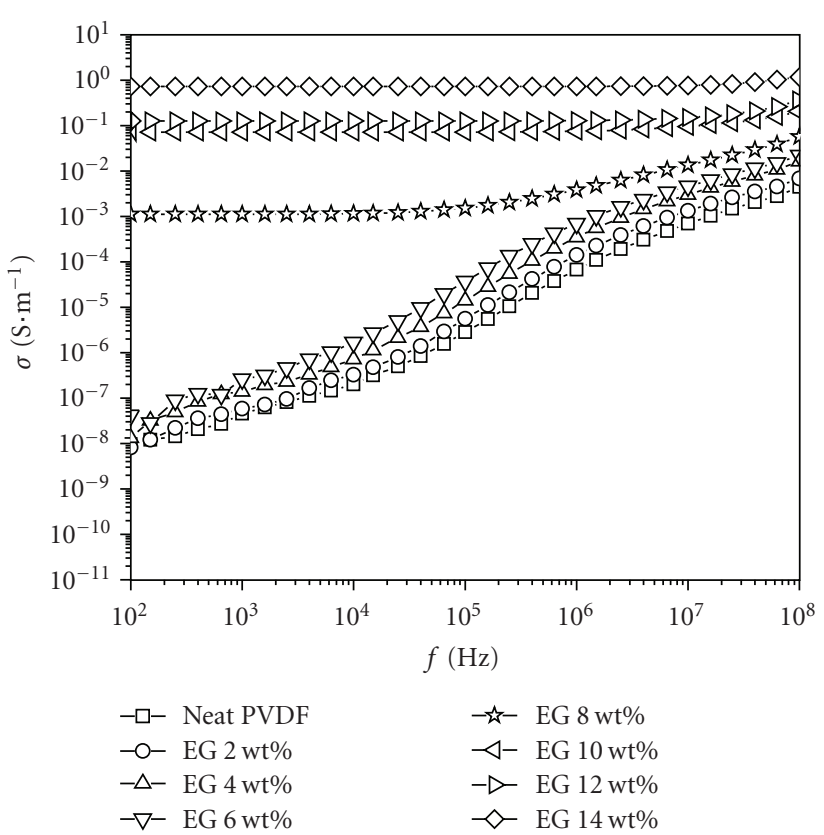

(a)

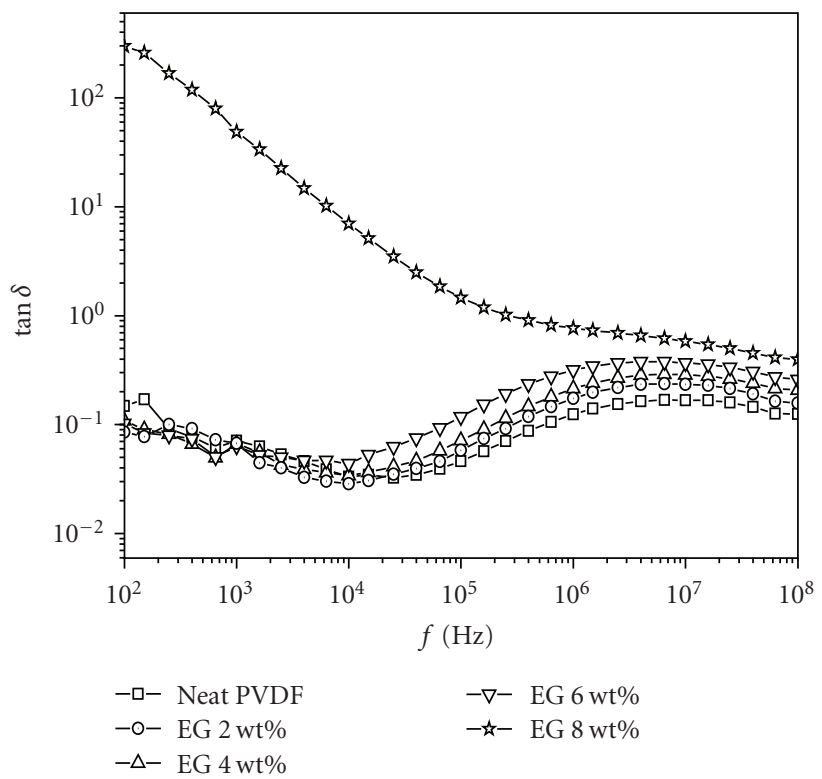

(b)

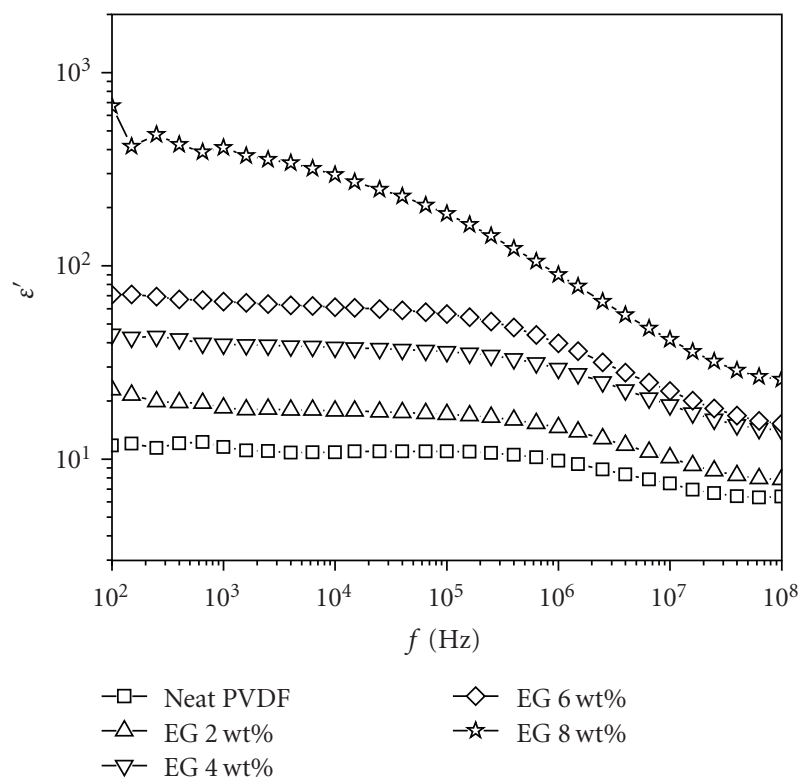

(c)

Figure 3: (a) Electrical conductivity, (b) loss tangent, and (c) dielectric constant of PVDF/EG composites as a function of frequency.

region in Figure 3(a). The effect of frequency highlights only in the high frequency range. Below percolation threshold, $\sigma_{\mathrm{DC}}$ is very small and can be neglect. Equation (4) equals to (2) and frequency prevails in the whole frequency region.

Figures 4(a) and 4(b) show the best fit of frequency dependent conductivity from (2) and (4) for representative PVDF/EG nanocomposites filled with EG contents of 6 and $8 \mathrm{wt} \%$ (the vincinity of $\Phi_{c}$ ). Accordingly, the variation in dielectric constant with frequency for these nanocomposites is fitted via (3) as shown in Figures 5(a) and 5(b), respectively. For the PVDF/6 wt\% EG nanocomposite, the electrical conductivity in the tested frequency region can be well described via (2) deriving a critical exponent $(u)$ of $1.05 \pm$ 0.02 as seen in Figure 4(a). Correspondingly, the dielectric constant of this composite is fitted via (3), but only high frequency region was used (Figure 5(a)), yielding a critical exponent $(v)$ of $0.21 \pm 0.01$. It is because, as mentioned above, the influence of frequency prevails or the dipolar polarizations of composites lose the response to electric fields in the high frequency region and the analyzed value in this region according to (3) is therefore much more precise to predict the frequency dependence of dielectric constant. For 


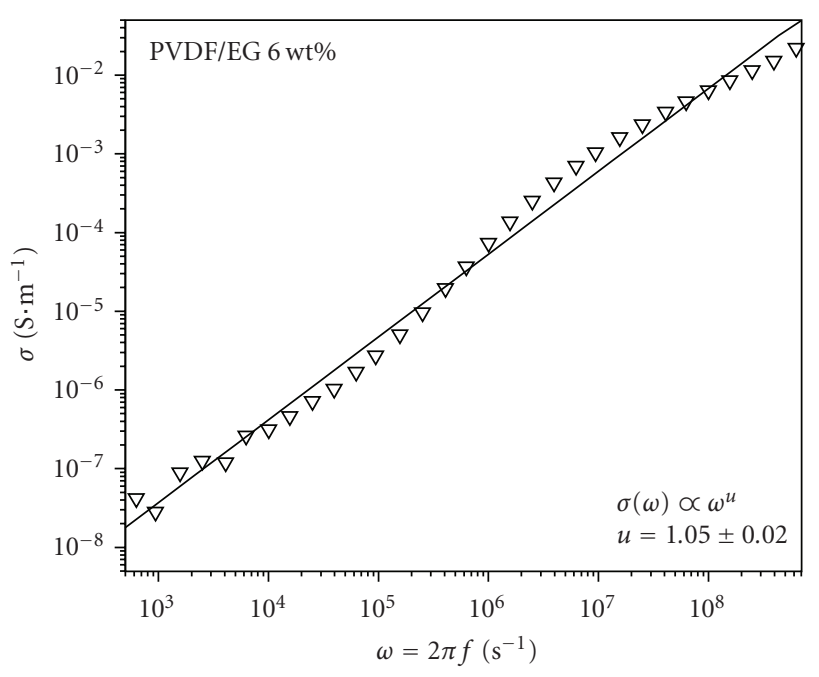

(a)

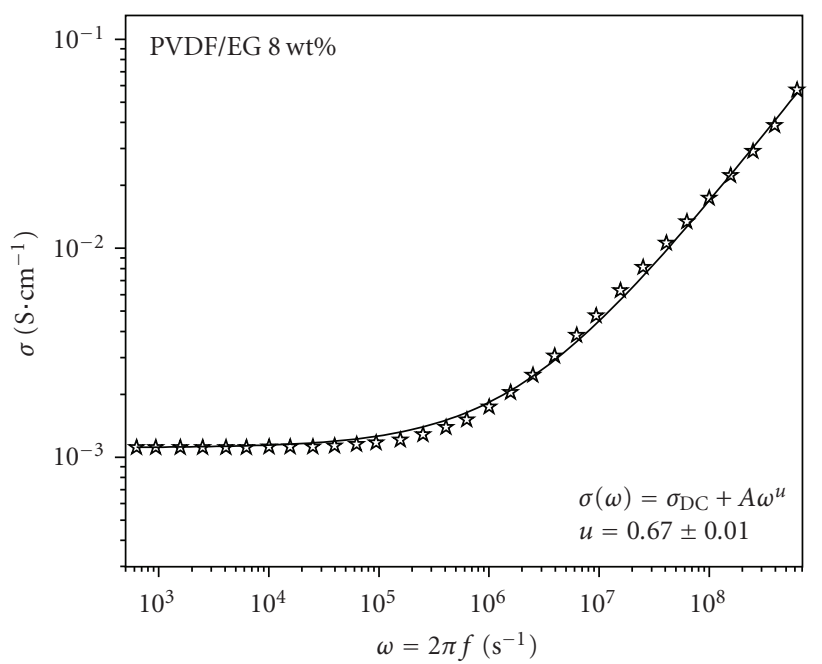

(b)

Figure 4: Best fit of frequency dependent conductivity for (a) PVDF/6 wt\% EG, and (b) PVDF/8 wt\% EG composites.

the PVDF/8 wt\% EG nanocomposite, the combined effect of $\sigma_{\text {DC }}$ and $f$ is clearly seen (Figure $4(\mathrm{~b})$ ). Above $f_{c}$ which is determined at which the AC conductivity reaches $110 \%$ of $\sigma_{\mathrm{DC}}$, that is, $\sigma_{f}=1.1 \sigma_{\mathrm{DC}}[23]$, the influence of frequency on conductivity became significant. The critical value $u$ derived from (4) is 0.67 . A sharp decrease of $u$ from 1.05 to 0.67 can be attributed to the large DC conductivity which results in the plateau region of the curve in the tested frequency ranges. Accordingly, the change in dielectric constant for PVDF/8 wt $\%$ EG composite shows much more dependant in the tested frequency region and the derived critical value $v=0.24$ which a little larger than that of PVDF/ 6 wt $\%$ EG composite.

For composites below $\Phi_{c}$, the $\sigma_{\mathrm{DC}}, \varepsilon_{\mathrm{DC}}$, can be determined from the $\sigma_{\mathrm{AC}}$ versus $f$ and $\varepsilon^{\prime}$ versus $f$ responses by simply extrapolating to $f=0 \mathrm{~Hz}$, respectively. Correspondingly, the temperature dependant parameter $A$ can

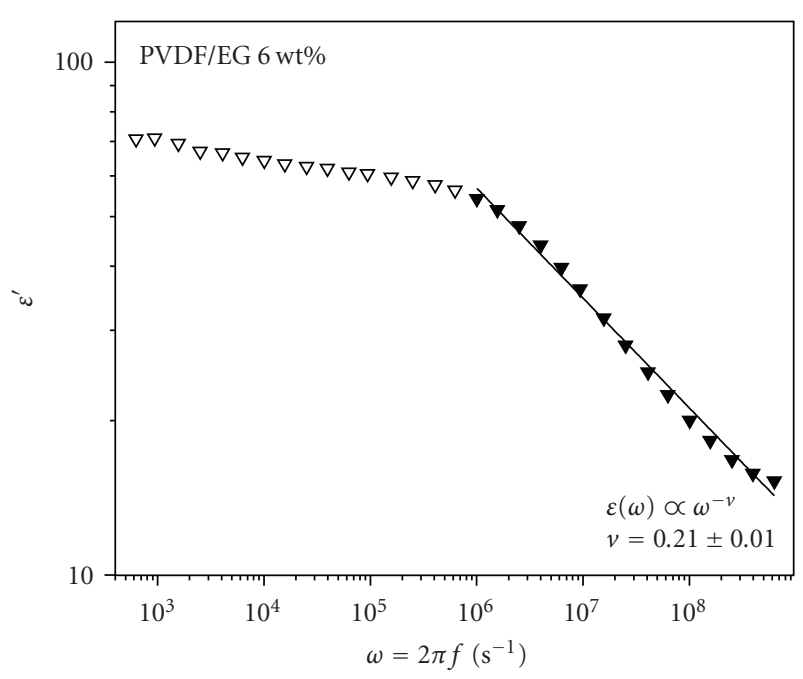

(a)

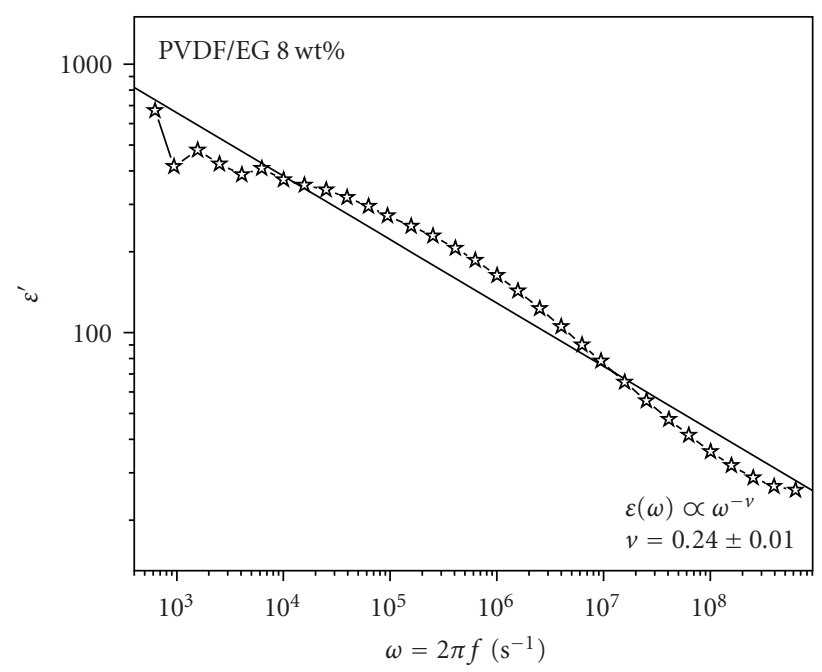

(b)

Figure 5: Best fit of frequency dependent dielectric constant for (a) PVDF/6 wt $\%$ EG and (b) PVDF/8 wt\% EG composites according to (3).

be obtained by applying the AC universal law conversely (equation (4)). The $\sigma_{\mathrm{DC}}, \varepsilon_{\mathrm{DC}}, u, v, A$ and $f_{c}$ of nanocomposites investigated are listed in Table 2 . The value of $u+$ $v$ for PVDF/6, $8 \mathrm{wt} \%$ EG composites are 1.26 and 0.91 , respectively. The deviance from theoretical value $(u+v=1)$ at $\Phi_{c}$ as presented above indicates the discrepancy of the use of percolation theory to interpret the real composites system with filler content far away from the percolation threshold.

3.2. DC Electrical Performance. To further discuss the charge transport of composites, we herein present their DC electrical performances. The plots of $\sigma_{\mathrm{DC}}$ and $\varepsilon_{\mathrm{DC}}$ (Table 2) versus EG volume content are depicted in Figures 6 and 7. A sharp increase in electrical conductivity $\left(\sim 10^{-1} \mathrm{~S} \cdot \mathrm{m}^{-1}\right)$ can be readily seen in Figure 6 when the EG content reaches $6.33 \mathrm{vol} \%(8 \mathrm{wt} \%)$. This is over eight orders of magnitude higher than that of pure PVDF $\left(3.26 \times 10^{-11} \mathrm{~S} \cdot \mathrm{m}^{-1}\right)$. The 
TABLE 2: Parameters depicting the frequency dependence of electrical properties of PVDF/EG composites.

\begin{tabular}{lcccccc}
\hline$\Phi(\mathrm{wt} \% / \mathrm{vol} \%)$ & $\sigma_{\mathrm{DC}}\left(\mathrm{S} \cdot \mathrm{m}^{-1}\right)$ & $f_{c}(\mathrm{~Hz})$ & $u$ & $A$ & $\varepsilon_{\mathrm{DC}}$ \\
\hline $0 / 0$ & $3.26 \mathrm{E}-11$ & - & $1.03 \pm 0.02$ & $4.06 \mathrm{E}-12$ & 12.7 & - \\
$2 / 1.56$ & $3.63 \mathrm{E}-11$ & - & $1.06 \pm 0.02$ & $3.55 \mathrm{E}-12$ & 24.3 & - \\
$4 / 3.14$ & $8.38 \mathrm{E}-11$ & - & $1.06 \pm 0.02$ & $8.40 \mathrm{E}-12$ & 48.8 & - \\
$6 / 4.73$ & $1.78 \mathrm{E}-10$ & - & $1.05 \pm 0.02$ & $1.41 \mathrm{E}-11$ & 82.3 & $0.21 \pm 0.01$ \\
$8 / 6.33$ & $1.07 \mathrm{E}-3$ & $4.0 \mathrm{E}+4$ & $0.67 \pm 0.01$ & $6.88 \mathrm{E}-8$ & $-1.18 \mathrm{E}-8$ & - \\
$10 / 7.95$ & 0.072 & $2.5 \mathrm{E}+6$ & - & $6.12 \mathrm{E}-11$ & - & - \\
$12 / 9.59$ & 0.127 & $6.5 \mathrm{E}+6$ & - & $3.37 \mathrm{E}-10$ & - \\
$14 / 11.24$ & 0.729 & $1.6 \mathrm{E}+7$ & - & - & - \\
\hline
\end{tabular}

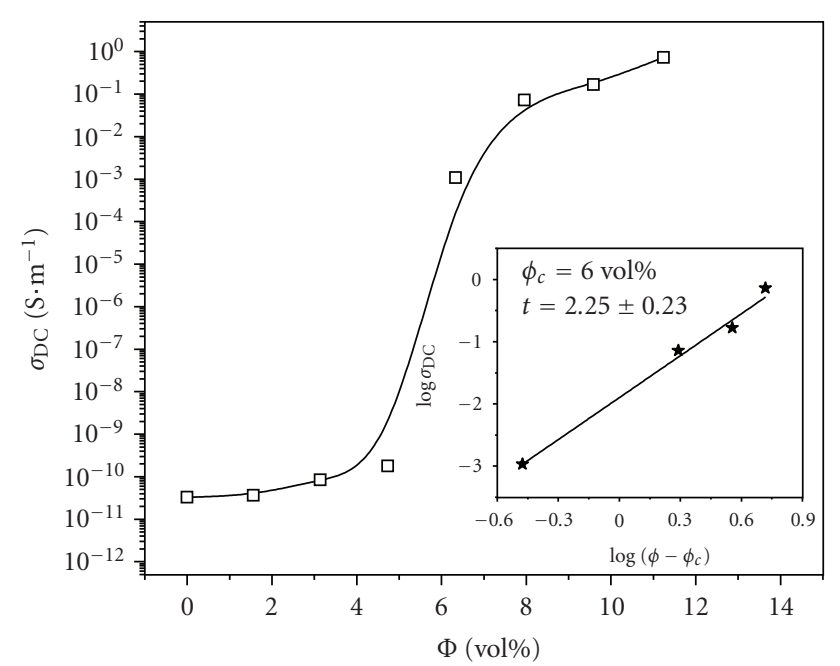

FIGURE 6: DC conductivity as a function of EG concentration.

conductivity levels off above 7.95 vol\% (10 wt $\%)$ due to the formation of entire conducting network in polymer matrix.

The conductivity generally follows the universal scaling law near the percolation concentration:

$$
\sigma_{m}=\sigma_{f}\left(\phi-\phi_{c}\right)^{t} \quad \text { for } \Phi>\Phi_{c}
$$

where $\sigma_{m}$ is the conductivity of composite, $\sigma_{f}$ is the conductivity of filler, $\Phi$ is the volume fraction of filler, $\Phi_{c}$ the percolation threshold and $t$ the critical exponent. It can be seen from the inset of Figure 6 that the plot of $\log \sigma$ versus $\log \left(\Phi-\Phi_{c}\right)$ yields the best-fit line with a percolation threshold of $6 \mathrm{vol} \%(\sim 7.6 \mathrm{wt} \%)$ and a critical exponent of $t=2.25$. For comparison, the percolation threshold of melt blended PVDF/MWCNT system is found to be $2-2.5 \mathrm{wt} \%[24,25]$. The low percolation threshold of the PVDF/MWCNT system can be attributed to the large aspect-ratio of CNTs. Since EG with worm-like morphology exhibits lower aspect ratio than CNTs, thus the percolation threshold of PVDF/EG system is much higher than that of the PVDF/MWCNT system. It is noted that the processing route for fabricating PVDF/EG nanocomposites also can affect their percolation threshold. In general, solution blending method tends to yield lower percolation threshold than the

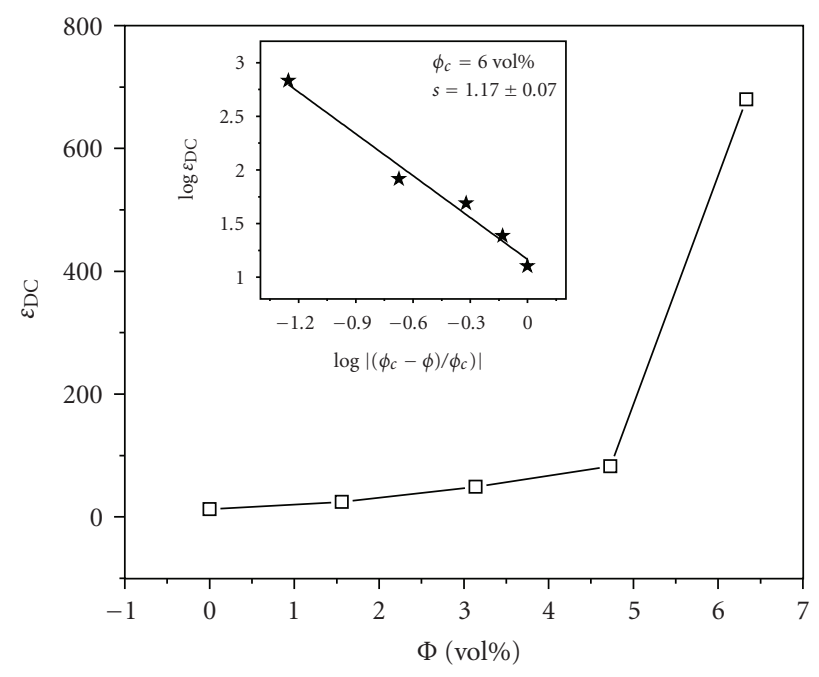

FIGURE 7: DC dielectric constant as a function of EG concentration.

melt blending process. Furthermore, the structure of EG can be further exfoliated to independent graphite nanoplatelets by subjecting to sonication. In this respect, the percolation threshold of PVDF/exfoliated graphite nanocomposites reduces considerably to 1 vol\% [14].

In the present study, the value of critical exponent is slightly higher than universal values $(1.6 \sim 2)$ reported for three dimensional randomly dispersed system [26]. It is recognized that tunneling conduction in real composites yields nonuniversal critical exponent values [27-30]. Recognizing the fact that the EG nanoplatelets are coated with thin PVDF film, thus tunneling conduction between nanoplatelets prevails above percolation concentration.

Similarly, the variation of dielectric constant in the neighborhood of $\Phi_{c}$ also follows the scaling law:

$$
\varepsilon_{m}=\varepsilon_{p}\left|\frac{\phi_{c}-\phi}{\phi_{c}}\right|^{-s} \quad \text { for } \Phi<\Phi_{c}
$$

where $\varepsilon_{p}$ is the dielectric constant of PVDF, $\varepsilon_{m}$ is the dielectric constant of composites, and $s$ is the critical exponent. Figure 7 shows the variation of dielectric constant with EG content for the PVDF/EG nanocomposites. A sharp increase in dielectric constant $(\varepsilon=680)$ is found when 


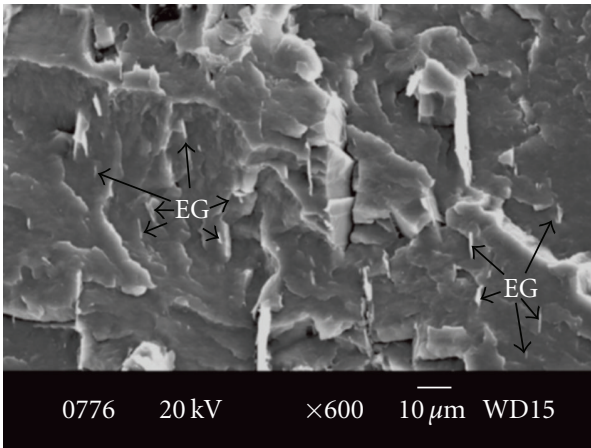

(a)

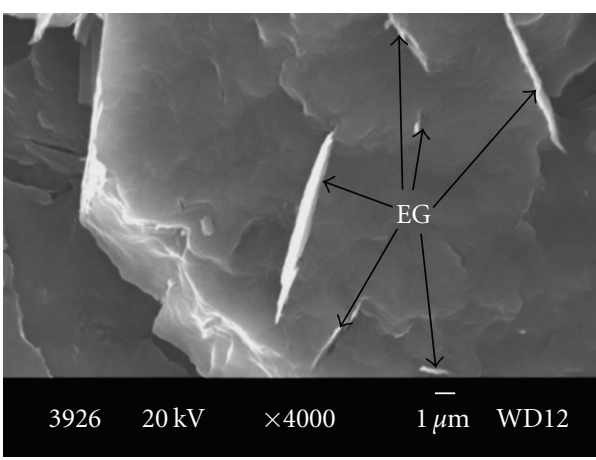

(b)

FIgURE 8: (a) Low and (b) large magnification SEM images of PVDF/6 wt $\%$ EG composites.

EG content reaches $6.33 \mathrm{vol} \%$ ( $8 \mathrm{wt} \%)$. It is nearly 70 times higher than that of neat PVDF matrix. Linear regression fit of experimental data using (6) yields a critical exponent of $s=1.17$ and $\Phi_{c}$ of $6 \mathrm{vol} \%$ (inset of Figure (7)). Such high dielectric constant at percolation concentration can be attributed to the minicapacitor effect. The minicapacitors consist of various graphite nanoplatelets separated by a thin insulating polymer matrix. This high dielectric behavior can be utilized to create high charge-storage devices [31].

The electrical properties of composites generally depend on the particle size, dispersion state and geometry of conducting fillers as well as the property of host polymer. Figures $8(\mathrm{a})$ and $8(\mathrm{~b})$ are SEM images of the resulting PVDF/6 wt\% EG composite. Most EG nanoplalets of large aspect-ratios can be seen to disperse homogeneously in PVDF matrix. From the literature, conducting nanofillers with large surface areas generally enhance the electrical transport in polymer composites [32]. Figure 9 shows the XRD patterns of representative composites. The peaks located at $18.5^{\circ}$ and $19.9^{\circ}$ can be assigned to the (020) and (110) reflections of $\alpha$-PVDF (inset). The graphite characteristic (002) peak can be readily seen at $2 \theta=26.6^{\circ}$ and its intensity increases with increasing EG loading.

3.3. Biased Random Walk Approximation. The transport properties of the percolating system are formulated in terms of diffusion (random walk) within the filler clusters [27].

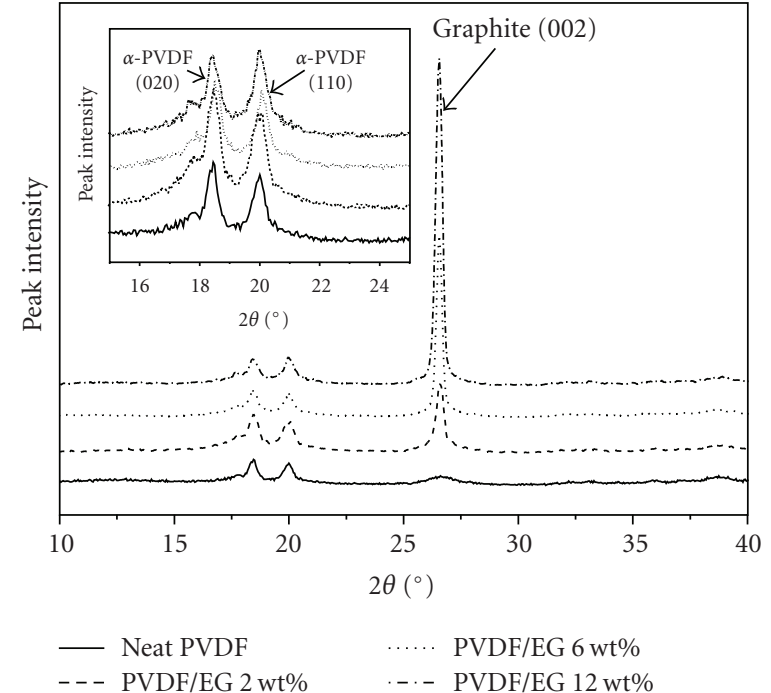

Figure 9: XRD curves for PVDF and representative PVDF/EG nanocomposites.

Accordingly, the correlation length $\xi$, defined as mean distance between the connectivity in the network can be used to describe the characteristic length scale of the system. At $\Phi_{c}$, the correlation length can be expressed as

$$
\xi \propto\left(\phi-\phi_{c}\right)^{-\gamma},
$$

where the $\gamma$ has the value of $4 / 3$ in two dimensions [23, 27] and 0.88 in three dimensions [21]. Recently, Kilbride et al. studied the AC and DC conducting behavior of polymer-carbon nanotube thin films [23]. They assumed that charge carriers undergo a biased random walk (BRW) along the conducting network with the relevant distance $L$ being traveled through the network. And then the frequency required for the carrier to travel a distance $L$ in one half period is given by $\omega \sim L^{-1 / a}$ with $a$ in the range of $0.5 \sim 1$.

Assuming that $\omega_{c}=\omega_{\xi}$, where $\omega_{\xi}$ is the frequency at which a carrier scans a distance equivalent to the correlation length $\xi$. Then, $\omega_{\xi}$ can be expresses as follows:

$$
\omega_{\xi} \propto \xi^{-1 / a} .
$$

Combining (6) and (7) and $\omega=2 \pi f$, the critical frequency can be expressed as

$$
f_{c} \propto\left(\phi-\phi_{c}\right)^{\gamma / a}
$$

The critical frequency $f_{c}$ for composites containing 8$14 \mathrm{wt} \%$ of EG listed in Table 2 is plotted against $\left(\Phi-\Phi_{c}\right)$ on a log-log scale as shown in Figure 10. It is clear that $f_{c}$ versus $\Phi-\Phi_{c}$ of PVDF/EG composites follow the power law relation quite well. From the slope of linear fit straight line, the exponent $\gamma / a$ is found to be 2.17. It has also been reported that $f_{c}$ has the relationship with $\sigma_{\mathrm{DC}}$ above $\Phi_{c}$ by a scaling power law [21, 29]:

$$
f_{c} \propto \sigma_{\mathrm{DC}}^{b}
$$




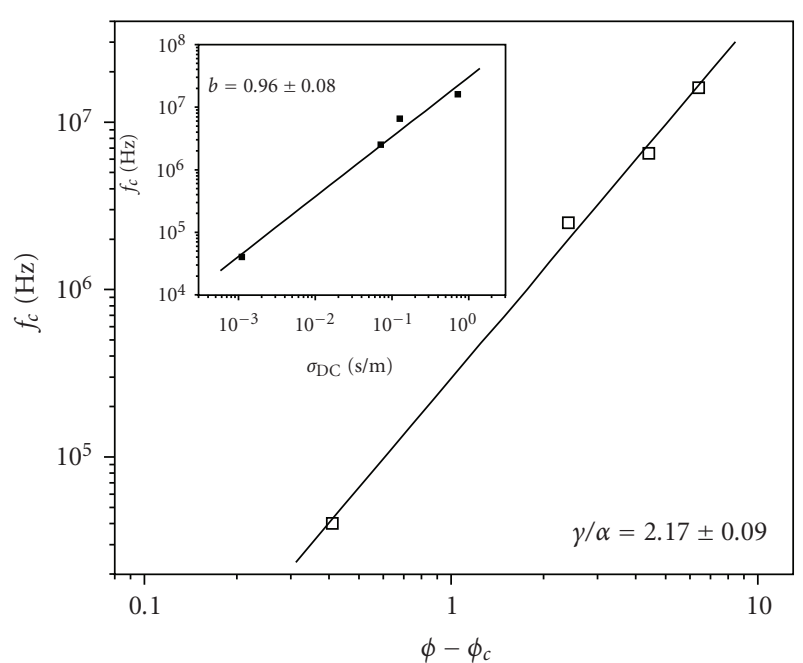

Figure 10: Log-log plot of critical frequency $f_{c}$ versus $\left(\Phi-\Phi_{c}\right)$ for PVDF/EG nanocomposites. The inset displays the relationship between $f_{c}$ and $\sigma_{\mathrm{DC}}$.

As can be seen from the inset of Figure 10, the experimental data well obeys this equation with an exponent $b=0.96$.

From (9) and (10), the relation $\sigma_{\mathrm{DC}} \propto\left(\Phi-\Phi_{c}\right)^{\gamma / a b}$ can be deduced, and this corresponds to the percolation conductivity as given in (5). Accordingly, $t=\gamma / a b=2.17 / 0.96$ $=2.26$. This value is very close to 2.25 directly obtained from the classical percolating equation as mentioned above. In this regard, the biased random walk approach and the assumption of $\omega_{c} \equiv \omega_{\xi}$ well describes the conductivity of percolating PVDF/EG system.

3.4. Effect of Temperature. Figure 11 illustrates the variation of resistivity (reciprocal of conductivity) of these nanocomposites, normalized to that at $30^{\circ} \mathrm{C}$, with increasing of temperature. The normalized resistivity of composite specimens increases slowly with increasing temperature up to the onset melting point $\left(T_{m}\right)$ of PVDF, follows by a sharp increase near peak melting temperature $\left(T_{m} \approx\right.$ $\left.160^{\circ} \mathrm{C}\right)$. The increase in resistivity for the PVDF/EG $8 \mathrm{wt} \%$ composite near the melting point is more than three orders of magnitude. This behavior is known as the positive temperature coefficient (PTC) effect commonly found in conducting filler/semicrystalline polymer composites [3337]. Above $T_{m}$, the normalized resistivity decreases markedly, referring to as the negative temperature coefficient (NTC) effect. Very recently, Ansari and Giannelis also reported the occurrence of NTC effect in the PVDF/EG system [38]. The exact mechanisms of PTC effect are complicated. Most researchers believe that the volume expansion of the matrix contribute to the abrupt increase of resistivity $[33,35]$.

The effect of temperature on dielectric constant for representative PVDF/8 wt\% EG composite at several frequencies is shown in Figure 12. The dielectric constant increases with decreasing frequency, showing consistent frequency dependence of dielectric constant (Figure 3(c)). In addition, the dielectric constant shows a plateau in low temperature

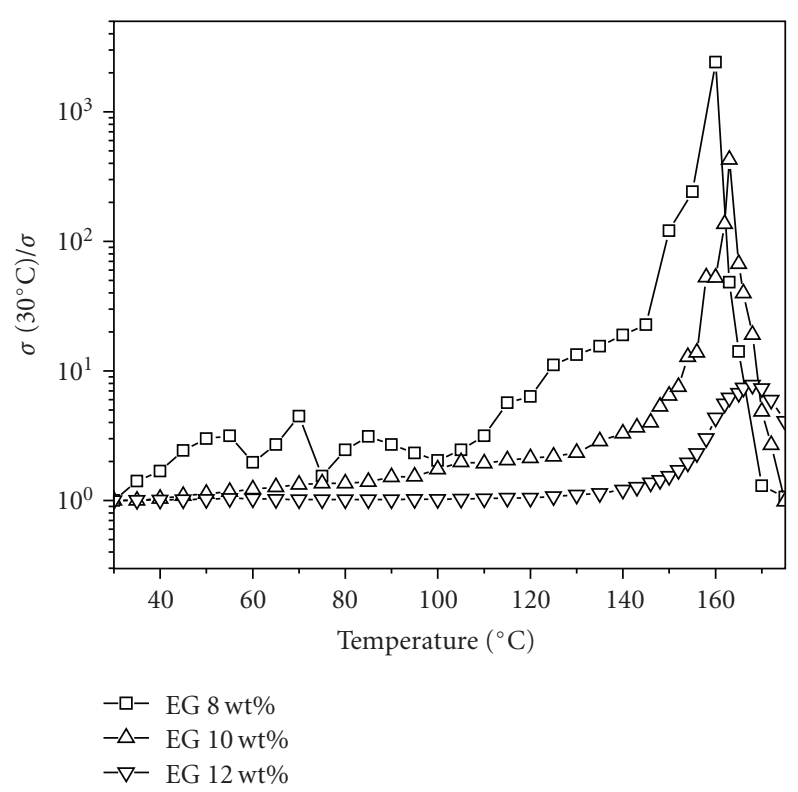

FIGURE 11: Variation of normalized resistivity with temperature.

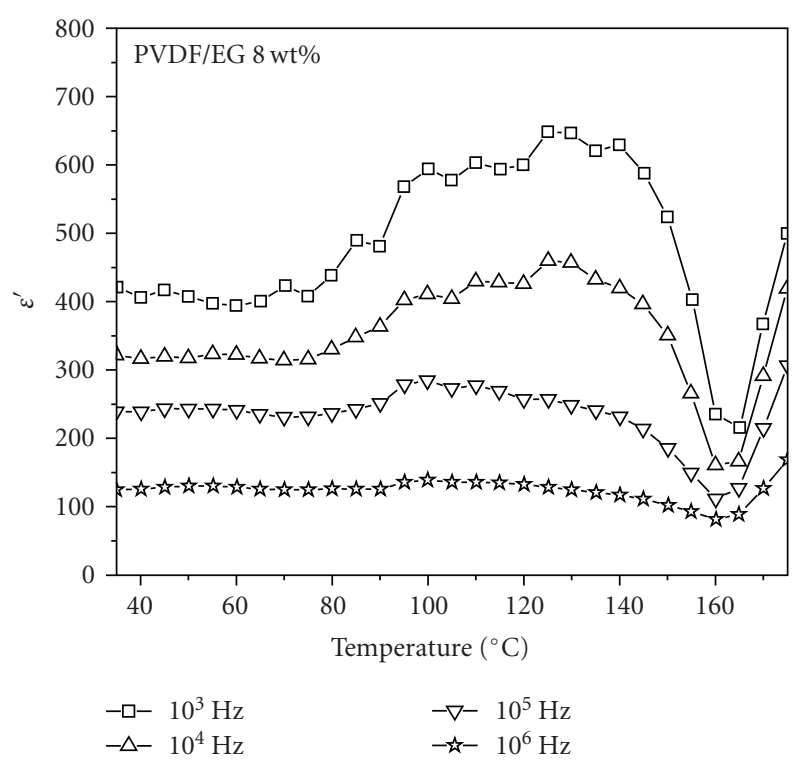

FIGURE 12: Dielectric constant as a function of frequency at various temperatures for PVDF/8 wt\% EG nanocomposite.

region $\left(T<80^{\circ} \mathrm{C}\right)$, while a broad peak emerges in temperature range of $80-140^{\circ} \mathrm{C}$. This behavior is particularly apparent for the composite tested at $10^{3}$ and $10^{4} \mathrm{~Hz}$, and associated with the $\alpha$-relaxation process of crystalline PVDF [39]. Above $140^{\circ} \mathrm{C}$, the dielectric constant decreases and exhibits an apparent minimum near the melting point of composites.

\section{Conclusions}

PVDF/EG composites were fabricated via direct melt compounding process. The electrical conductivity and dielectric 
properties of PVDF/EG composites were measured in a wide range of frequencies from $10^{2}$ to $10^{8} \mathrm{~Hz}$ at various temperatures. The results showed that the AC conductivity $(\sigma)$ and dielectric constant $\left(\varepsilon^{\prime}\right)$ of the PVDF/EG composites near the percolation threshold were frequency dependent and obeyed the power law relations $\left(\sigma \propto \omega^{u}\right.$ and $\varepsilon^{\prime} \propto$ $\left.\omega^{-v}\right)$. The value of critical exponents $u+v$ for PVDF/6 wt $\%$ EG and PVDF/8 wt\% EG composites were determined to be 1.26 and 0.91 , respectively. The DC conductivity and dielectric constant extrapolated from AC results followed with the percolation scaling law, yielding a percolation threshold $\Phi_{c}=6$ vol $\%$ and critical exponents $t=2.25$ and $s=1.17$, respectively. The introduction of biased random walk approach well described the conductivity of percolating PVDF/EG system. Finally, percolating PVDF/EG composites displayed positive temperature coefficient effect near the melting point of composites. The dielectric constant showed a broad peak in temperature range of $80-140^{\circ} \mathrm{C}$ which denoted as the $\alpha$-relaxation process of crystalline PVDF.

\section{References}

[1] K. Kalaitzidou, H. Fukushima, and L. T. Drzal, "Mechanical properties and morphological characterization of exfoliated graphite-polypropylene nanocomposites," Composites Part A, vol. 38, no. 7, pp. 1675-1682, 2007.

[2] F. M. Uhl, Q. Yao, H. Nakajima, E. Manias, and C. A. Wilkie, "Expandable graphite/polyamide-6 nanocomposites," Polymer Degradation and Stability, vol. 89, no. 1, pp. 70-84, 2005.

[3] W. Zheng and S.-C. Wong, "Electrical conductivity and dielectric properties of PMMA/expanded graphite composites," Composites Science and Technology, vol. 63, no. 2, pp. 225-235, 2003.

[4] G. Chen, W. Weng, D. Wu, and C. Wu, "PMMA/graphite nanosheets composite and its conducting properties," European Polymer Journal, vol. 39, no. 12, pp. 2329-2335, 2003.

[5] P. Pötschke, S. M. Dudkin, and I. Alig, "Dielectric spectroscopy on melt processed polycarbonate-multiwalled carbon nanotube composites," Polymer, vol. 44, no. 17, pp. 5023 5030, 2003.

[6] Z. Ounaies, C. Park, K. E. Wise, E. J. Siochi, and J. S. Harrison, "Electrical properties of single wall carbon nanotube reinforced polyimide composites," Composites Science and Technology, vol. 63, no. 11, pp. 1637-1646, 2003.

[7] A. Boudenne, L. Ibos, M. Fois, J. C. Majesté, and E. Géhin, "Electrical and thermal behavior of polypropylene filled with copper particles," Composites Part A, vol. 36, no. 11, pp. 15451554, 2005.

[8] J.-W. Kim, W.-J. Cho, and C.-S. Ha, "Morphology, crystalline structure, and properties of poly(vinylidene fluoride)/silica hybrid composites," Journal of Polymer Science, Part B, vol. 40, no. 1, pp. 19-30, 2002

[9] M. Wang, J.-H. Shi, K. P. Pramoda, and S. H. Goh, "Microstructure, crystallization and dynamic mechanical behaviour of poly(vinylidene fluoride) composites containing poly(methyl methacrylate)-grafted multiwalled carbon nanotubes," Nanotechnology, vol. 18, no. 23, Article ID 235701, 7 pages, 2007.

[10] D. Shah, P. Maiti, E. Gunn, et al., "Dramatic enhancements in toughness of polyvinylidene fluoride nanocomposites via nanoclay-directed crystal structure and morphology," Advanced Materials, vol. 16, no. 14, pp. 1173-1177, 2004.
[11] A. Almasri, Z. Ounaies, Y. S. Kim, and J. Grunlan, "Characterization of solution-processed double-walled carbon nanotube/ poly(vinylidene fluoride) nanocomposites," Macromolecular Materials and Engineering, vol. 293, no. 2, pp. 123-131, 2008.

[12] S. C. Tjong, G. D. Liang, and S. P. Bao, "Electrical behavior of polypropylene/multiwalled carbon nanotube nanocomposites with low percolation threshold," Scripta Materialia, vol. 57, no. 6, pp. 461-464, 2007.

[13] G. D. Liang and S. C. Tjong, "Electrical properties of percolative polystyrene/carbon nanofiber composites," IEEE Transactions on Dielectrics and Electrical Insulation, vol. 15, no. 1, pp. 214-220, 2008.

[14] F. He, S. Lau, H. L. Chan, and J. Fan, "High dielectric permittivity and low percolation threshold in nanocomposites based on poly(vinylidene fluoride) and exfoliated graphite nanoplates," Advanced Materials, vol. 21, no. 6, pp. 710-715, 2009.

[15] Y. Li, R. K. Y. Li, and S. C. Tjong, "Fabrication and properties of PVDF/expanded graphite nanocomposites," E-Polymers, no. 19, pp. 1-13, 2009.

[16] H. Quan, B.-Q. Zhang, Q. Zhao, R. K. K. Yuen, and R. K. Y. Li, "Facile preparation and thermal degradation studies of graphite nanoplatelets (GNPs) filled thermoplastic polyurethane (TPU) nanocomposites," Composites Part A, vol. 40, no. 9, pp. 1506-1513, 2009.

[17] J. Meyer, "Glass transition temperature as a guide to selection of polymers suitable for PTC materials," Polymer Engineering and Science, vol. 13, no. 6, pp. 462-468, 1973.

[18] C.-W. Nan, "Physics of inhomogeneous inorganic materials," Progress in Materials Science, vol. 37, no. 1, pp. 1-116, 1993.

[19] G. C. Psarras, E. Manolakaki, and G. M. Tsangaris, "Dielectric dispersion and ac conductivity in-Iron particles loadedpolymer composites," Composites Part A, vol. 34, no. 12, pp. 1187-1198, 2003.

[20] H. Böttger and V. V. Bryksin, Hopping Conduction in Solids, Akademie, Berlin, Germany, 1985.

[21] S. Barrau, P. Demont, A. Peigney, C. Laurent, and C. Lacabanne, "DC and AC conductivity of carbon nanotubespolyepoxy composites," Macromolecules, vol. 36, no. 14, pp. 5187-5194, 2003.

[22] G. C. Psarras, "Hopping conductivity in polymer matrixmetal particles composites," Composites Part A, vol. 37, no. 10, pp. 1545-1553, 2006.

[23] B. E. Kilbride, J. N. Coleman, J. Fraysse, et al., "Experimental observation of scaling laws for alternating current and direct current conductivity in polymer-carbon nanotube composite thin films," Journal of Applied Physics, vol. 92, no. 7, pp. 40244030, 2002.

[24] Y. W. Nam, W. N. Kim, Y. H. Cho, et al., "Morphology and physical properties of binary blend based on PVDF and multiwalled carbon nanotube," Macromolecular Symposia, vol. 249250, pp. 478-484, 2007.

[25] S. M. Hong and S. S. Hwang, "Physical properties of thin PVDF/MWNT (multi-walled carbon nanotube) composite films by melt blending," Journal of Nanoscience and Nanotechnology, vol. 8, no. 9, pp. 4860-4863, 2008.

[26] S. Kirkpatrick, "Percolation and conduction," Reviews of Modern Physics, vol. 45, no. 4, pp. 574-588, 1973.

[27] D. Stauffer and A. Aharony, Introduction to Percolation Theory, Taylor \& Francis, London, UK, 1994.

[28] I. Balberg, "Tunneling and nonuniversal conductivity in composite materials," Physical Review Letters, vol. 59, no. 12, pp. 1305-1308, 1987. 
[29] J. C. Dyre and T. B. Schrøder, "Universality of AC conduction in disordered solids," Reviews of Modern Physics, vol. 72, no. 3, pp. 873-892, 2000.

[30] J. R. Macdonald, "Impedance spectroscopy," Annals of Biomedical Engineering, vol. 20, no. 3, pp. 289-305, 1992.

[31] L. Wang and Z.-M. Dang, "Carbon nanotube composites with high dielectric constant at low percolation threshold," Applied Physics Letters, vol. 87, no. 4, Article ID 042903, 3 pages, 2005.

[32] J. K. Nelson and J. C. Fothergill, "Internal charge behaviour of nanocomposites," Nanotechnology, vol. 15, no. 5, pp. 586-595, 2004.

[33] Y. Song, Y. Pan, Q. Zheng, and X.-S. Yi, "Electric selfheating behavior of graphite-filled high-density polyethylene composites," Journal of Polymer Science, Part B, vol. 38, no. 13, pp. 1756-1763, 2000.

[34] H. Xie, P. Deng, L. Dong, and J. Sun, "LDPE/carbon black conductive composites: influence of radiation crosslinking on PTC and NTC properties," Journal of Applied Polymer Science, vol. 85, no. 13, pp. 2742-2749, 2002.

[35] J. Fournier, G. Boiteux, G. Seytre, and G. Marichy, "Positive temperature coefficient effect in carbon black/epoxy polymer composites," Journal of Materials Science Letters, vol. 16, no. 20, pp. 1677-1679, 1997.

[36] X. He, L. Wang, and X. Chen, "PTC effect in carbon blackfilled ethylene-propylene-diene terpolymer systems," Journal of Applied Polymer Science, vol. 80, no. 9, pp. 1571-1574, 2001.

[37] X. J. He, J. H. Du, Z. Ying, H. M. Cheng, and X. J. He, "Positive temperature coefficient effect in multiwalled carbon nanotube/high-density polyethylene composites," Applied Physics Letters, vol. 86, no. 6, Article ID 062112, 3 pages, 2005.

[38] S. Ansari and E. P. Giannelis, "Functionalized graphene sheet-poly(vinylidene fluoride) conductive nanocomposites," Journal of Polymer Science, Part B, vol. 47, no. 9, pp. 888-897, 2009.

[39] Z.-M. Dang, W.-T. Yan, and H.-P. Xu, "Novel high-dielectricpermittivity poly(vinylidene fluoride)/polypropylene blend composites: the influence of the poly(vinylidene fluoride) concentration and compatibilizer," Journal of Applied Polymer Science, vol. 105, no. 6, pp. 3649-3655, 2007. 

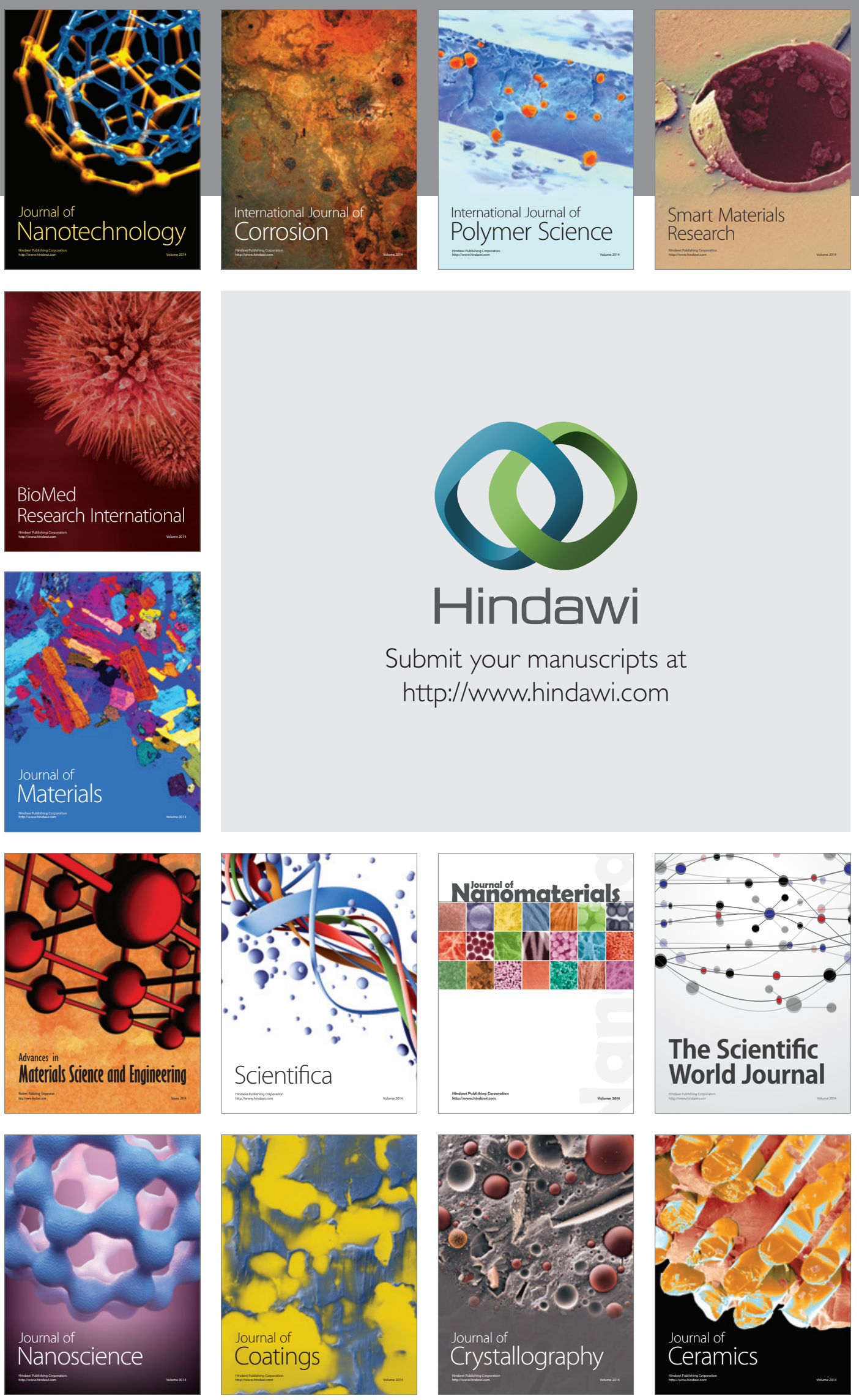

The Scientific World Journal

Submit your manuscripts at

http://www.hindawi.com

\section{World Journal}

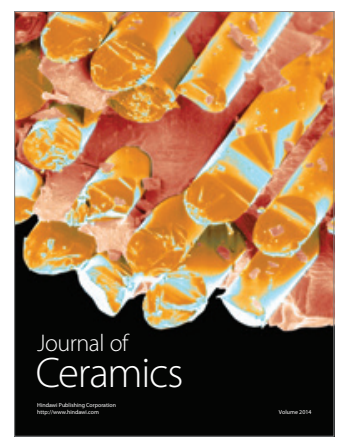

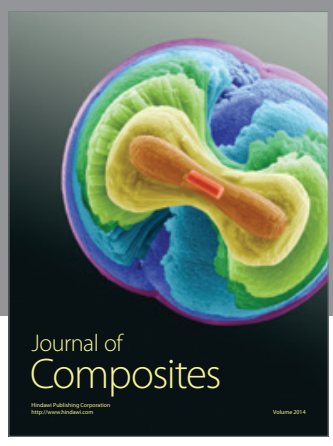
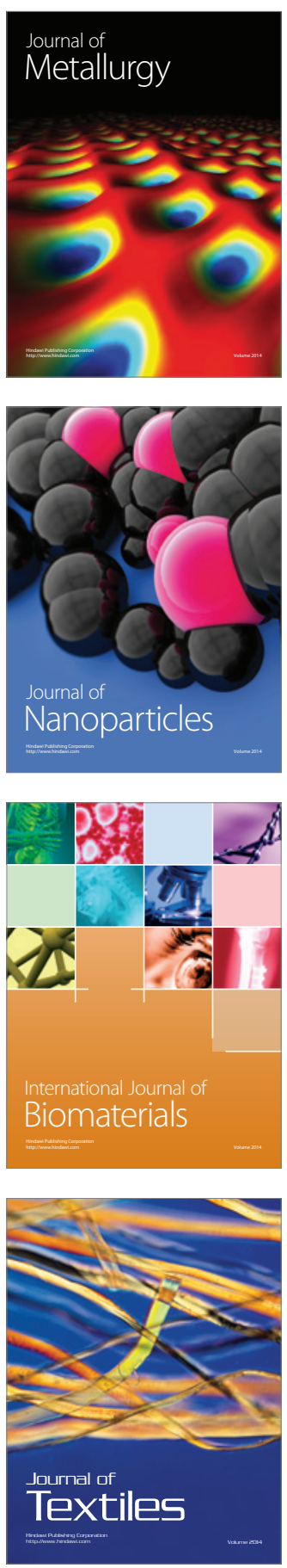